This copy is for your personal, non-commercial use only.

If you wish to distribute this article to others, you can order high-quality copies for your colleagues, clients, or customers by clicking here.

Permission to republish or repurpose articles or portions of articles can be obtained by following the guidelines here.

The following resources related to this article are available online at www.sciencemag.org (this information is current as of March 10, 2014 ):

Updated information and services, including high-resolution figures, can be found in the online version of this article at:

http://www.sciencemag.org/content/288/5466/640.full.html

This article cites $\mathbf{7 6}$ articles, 30 of which can be accessed free:

http://www.sciencemag.org/content/288/5466/640.full.html\#ref-list-1

This article has been cited by 285 article(s) on the ISI Web of Science

This article has been cited by 100 articles hosted by HighWire Press; see:

http://www.sciencemag.org/content/288/5466/640.full.html\#related-urls

This article appears in the following subject collections:

Biochemistry

http://www.sciencemag.org/cgi/collection/biochem 


\title{
Architecture of RNA Polymerase II and Implications for the Transcription Mechanism
}

\author{
Patrick Cramer, ${ }^{1}$ David A. Bushnell, ${ }^{1}$ Jianhua Fu, ${ }^{1}$ \\ Averell L. Gnatt, ${ }^{1}$ Barbara Maier-Davis, ${ }^{1}$ Nancy E. Thompson, ${ }^{2}$ \\ Richard R. Burgess, ${ }^{2}$ Aled M. Edwards, ${ }^{3}$ Peter R. David, ${ }^{1}$ \\ Roger D. Kornberg ${ }^{1 *}$
}

\begin{abstract}
A backbone model of a 10-subunit yeast RNA polymerase II has been derived from $x$-ray diffraction data extending to 3 angstroms resolution. All 10 subunits exhibit a high degree of identity with the corresponding human proteins, and 9 of the 10 subunits are conserved among the three eukaryotic RNA polymerases I, II, and III. Notable features of the model include a pair of jaws, formed by subunits Rpb1, Rpb5, and Rpb9, that appear to grip DNA downstream of the active center. A clamp on the DNA nearer the active center, formed by Rpb1, $\mathrm{Rpb} 2$, and Rpb6, may be locked in the closed position by RNA, accounting for the great stability of transcribing complexes. A pore in the protein complex beneath the active center may allow entry of substrates for polymerization and exit of the transcript during proofreading and passage through pause sites in the DNA.
\end{abstract}

RNA polymerase II (pol II), the central enzyme of gene expression, synthesizes all messenger RNA in eukaryotes. The intricate regulation of pol II transcription underlies cell growth and differentiation. The size and complexity of pol II befit this important role. The best characterized form of the enzyme, that from the yeast Saccharomyces cerevisiae, comprises 12 different polypeptides, with a total mass of about 0.5 megadaltons (MD) (Table 1). The human enzyme must be virtually identical, as the human genes for all subunits show a high degree of sequence conservation (Table 1), and at least 10 mammalian pol II genes can be substituted for their counterparts in yeast (1).

Pol II is the core of the transcription machinery. On its own, it can unwind the DNA double helix, polymerize RNA, and proofread the nascent transcript. In the presence of additional proteins, it assembles even larger initiation and elongation complexes, capable of promoter recognition and response to regulatory signals. A regulated initiation complex comprises pol II, five general transcription factors, and a multiprotein Mediator (24 ). It contains some 60 proteins, with a total

${ }^{1}$ Department of Structural Biology, Stanford University School of Medicine, Stanford, CA 94305-5126, USA. ${ }^{2}$ McArdle Laboratory for Cancer Research, University of Wisconsin, Madison, Madison, WI 53706, USA. ${ }^{3}$ Banting and Best Department of Medical Research, University of Toronto, Toronto, M5G 1L6, Canada.

*To whom correspondence should be addressed. Email: kornberg@stanford.edu mass of 3.5 MD. In transcription elongation complexes, Mediator and some of the general transcription factors are replaced by SII (TFIIS), Elongator, other elongation factors, and RNA processing proteins $(3,5,6)$.

Determination of molecular models for the pol II transcription machinery has so far been limited to a half dozen of the smallest proteins and protein fragments (7-17). Detailed structural studies of the larger proteins and multiprotein complexes, essential for understanding the mechanism and regulation of transcription, pose a more formidable challenge. We report here the x-ray analysis of a 10 -subunit yeast pol II. As nine of the subunits are conserved among RNA polymerases I, II, and III (18), our findings provide a basis for understanding the entire eukaryotic transcription machinery. They suggest roles for each of the many subunits and give insight into the remarkable features of the transcription mechanism.

Our investigation stemmed originally from the development of a yeast cell extract capable of accurately initiated pol II transcription (19) and the development of a general method of forming single-layer [two-dimensional (2D)] protein crystals (20). An active extract opened the way to the isolation of functional pol II (21), whereas the $2 \mathrm{D}$ crystallographic approach extended the reach of structure determination to such scarce, large, fragile multiprotein complexes. The first 2D crystallization trials gave crystals too small and too poorly ordered for structure determination (21). However, the ease and small amount of material required for $2 \mathrm{D}$ crystallization allowed its use as a structural assay to guide the preparation of pol II that would form better crystals. It soon emerged that heterogeneity of pol II, owing to substoichiometric levels of two small subunits, Rpb4 and $\mathrm{Rpb} 7$, was an impediment to crystallization. The problem was solved by the isolation of pol II from an $R P B 4$ deletion strain of yeast, yielding a "deletion" enzyme lacking both Rpb4 and Rpb7, which together account for only $8 \%$ of the mass of the wild-type protein. The deletion enzyme, unimpaired in transcription elongation and also fully active in transcription initiation when supplemented with the missing subunits (22), formed exceptionally large, well-ordered 2D crystals (23). Structures of pol II alone, and complexed with general transcription factors and nucleic acids, were determined by $3 \mathrm{D}$ reconstruction from electron micrographs of 2D crystals to about $15 \AA$ resolution (24-27). In the course of this work, it became apparent that even at the low protein concentration used for 2D crystallization, typically about $0.1 \mathrm{mg} / \mathrm{ml}$, there was a tendency of the crystals to grow epitaxially, adding additional layers in register with the first (23). This tendency was exploited by the use of $2 \mathrm{D}$ crystals as seeds for growing $3 \mathrm{D}$ crystals (28), which are now readily obtained by conventional methods as well.

X-ray diffraction from 3D crystals of pol II was initially undetectable. The problem proved to be oxidation. Maintenance of an inert atmosphere during the final stages of protein purification and throughout crystal growth, as well as improvements in crystallization conditions, enabled the collection of diffraction data to $3.5 \AA$ resolution (29). Because of the great size of the protein and unit cell, only large heavy atom clusters, such as an 18-tungsten-atom cluster, could be used for initial phase determination. The validity of the initial phases was shown by a close fit of the electron density map computed at $6 \AA$ resolution to the pol II map from 2D crystallography (29). There was only one deviation between the two maps, which was attributed to movement of a protein domain, suggested to clamp nucleic acid in a transcribing complex (29).

With a $6 \AA$ phase set, it should have been possible to locate individual heavy atoms in isomorphous derivatives and to extend structure determination to higher resolution. There were, however, three major obstacles. First, diffraction to $3.5 \AA$ resolution could not be obtained reproducibly. Second, the crystals were nonisomorphous, varying by as much as $10 \AA$ in one dimension of the unit cell. Very few crystals could be derivatized and matched with an isomorphous native crystal. Because of the low abundance of pol II, approximately 10,000 liters of cell culture had to be processed to obtain the $6 \AA$ electron density map, and far more would have been required for extension to high resolution. The final obstacle was that heavy atom compounds commonly used for protein 


\section{RESEAR CHARTICLES}

phase determination destroyed diffraction from the crystals.

A crystallographic backbone model for RNA polymerase II. These difficulties were overcome in the present work by a soaking procedure that shrank the crystals to an apparent minimum of the variable unit cell dimension (30). The resulting crystals were isomorphous and diffracted isotropically to $3.0 \AA$ resolution (31). Because the improved crystals were nonisomorphous with the original crystals, initial phases were redetermined by multiple anomalous dispersion (MAD) with a six-tantalumatom cluster derivative, which showed a single peak in difference Pattersons (Fig. 1) (32). These phases sufficed to reveal individual heavy atoms in other crystals by means of cross-difference Fouriers (Fig. 1) (33). An extensive search identified nonstandard mononuclear heavy atom compounds that gave useful derivatives (Table 2) (34). Phases were determined by multiple isomorphous replacement with anomalous scattering (MIRAS) from 10 data sets, ranging from 4.0 to $3.1 \AA$ resolution (Table 2) (35). The resulting molecular envelope was in good agreement with that previously obtained at $6 \AA$ resolution (29). After solvent flattening, an electron density map was obtained that revealed the course of the polypeptide chain and many amino acid side chains (Fig. 2) (36).

Available structures of pol II subunits and subunit fragments, comprising $14 \%$ of all pol II amino acid residues, were manually fit into the electron density (37). The complete structures of yeast Rpb5 and Rpb8 were used, whereas structures of Escherichia coli and archaebacterial homologs of yeast Rpb3, 6, 9, 10, and 11 were truncated to the conserved regions (Table
1). In all cases, a unique fit of the subunit fold to regions of the electron density map was observed. Subunit placement was facilitated by the location of eight zinc ions, revealed by a zinc anomalous difference Fourier (Fig. 1 and Table 1). Most parts of the yeast subunits missing from the homologous proteins could be modeled as polyalanine into adjacent regions of electron density. The remaining density, about $70 \%$ of the total volume, was attributed to the two large subunits, Rpb1 and Rpb2, with a
A

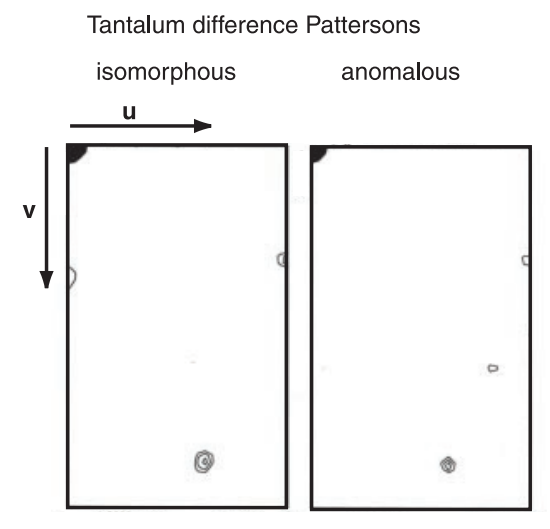

minor contribution from the smallest subunit, Rpb12. It was modeled as polyalanine fragments, with the use of standard secondary structure elements wherever possible. Combination of phases from MIRAS and an initial polyalanine model resulted in an improved map, which allowed adjustment and extension of the model (38). The polyalanine fragments were assigned to Rpb1 or Rpb2 on the basis of (i) the location of the active-site metal bound by Rpb1 (see below); (ii) two zinc-binding motifs in the $\mathrm{NH}_{2}$ -
B

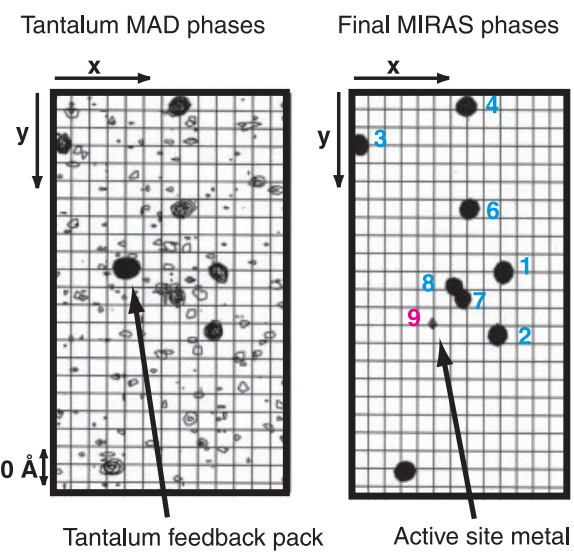

Fig. 1. Localization of heavy atoms. (A) Harker sections of isomorphous and anomalous difference Patterson maps of the tantalum cluster derivative (Table 2). A single peak at the same position in the two maps is observed. Heights of the Harker peaks in the isomorphous and anomalous difference Pattersons were $6 \sigma$ and $5 \sigma$, respectively. The resolution range of the data used is 40 to $5.5 \AA$. The contour levels are $3 \sigma$ (background) and $1 \sigma$ (steps). (B) Anomalous difference Fourier calculated with native data collected at the zinc anomalous peak energy using initial tantalum MAD phases (left) and final MIRAS phases (right). The projection of one asymmetric unit along the $z$ axis is shown for tantalum and MIRAS phases at a contour level of $3 \sigma$ and $7 \sigma$, respectively, with $1 \sigma$ steps. The eight strong peaks correspond to structural zinc atoms (Table 1). The ninth peak corresponds to the active site metal and likely arises from partial replacement of magnesium by zinc.

Table 1. Yeast RNA polymerase II subunits.

\begin{tabular}{|c|c|c|c|c|c|c|c|c|c|c|c|c|}
\hline \multirow[b]{2}{*}{ Subunit } & \multirow{2}{*}{$\begin{array}{l}\text { Mass } \\
(\mathrm{kD})\end{array}$} & \multirow{2}{*}{$\begin{array}{l}\text { Residues in } \\
\text { sequence }\end{array}$} & \multirow{2}{*}{$\begin{array}{l}\text { Identity to } \\
\text { human (\%) }\end{array}$} & \multicolumn{6}{|c|}{ Structure used in modeling } & \multirow{2}{*}{$\begin{array}{l}\text { Residues in } \\
\text { model (\%) }\end{array}$} & \multirow[b]{2}{*}{ Zinc site $(\sigma)^{2}$} & \multirow{2}{*}{$\begin{array}{c}\text { Surface } \\
\text { cysteines }^{3}\end{array}$} \\
\hline & & & & Organism & Protein & Method & $\begin{array}{l}\text { PDB } \\
\text { code }\end{array}$ & $\begin{array}{l}\text { Refer- } \\
\text { ence }\end{array}$ & $\begin{array}{l}\text { Conserved } \\
\text { residues }\end{array}$ & & & \\
\hline Rpb1 & 191.6 & $\begin{array}{c}1733 \\
(1449)^{5}\end{array}$ & 52 & & & & & & & $1213(84)^{5}$ & $\begin{array}{c}\mathrm{Zn6} \text { (23.2), } \\
\mathrm{Zn8}(19.3), \\
\text { "Zn9" (9.8) }\end{array}$ & \\
\hline Rpb2 & 138.8 & 1224 & 61 & & & & & & & $949(78)$ & $\mathrm{Zn7}(23.1)$ & \\
\hline Rpb3 & 35.3 & 318 & 46 & E. coli & $\alpha$ & X-ray & $1 b d f$ & $(69)$ & $\begin{array}{l}8-69,162- \\
180,233- \\
251\end{array}$ & $264(83)$ & $\operatorname{Zn} 2(30.2)$ & Cys207 \\
\hline Rpb4 & 25.4 & 221 & 30 & & & & & & & & & \\
\hline Rpb5 & 25.1 & 215 & 45 & S. cerevisiae & Rpb5 & X-ray & $1 \mathrm{dzf}$ & $(47)$ & & 211 (98) & & Cys83 \\
\hline Rpb6 & 17.9 & 155 & 59 & Human & RPABC14.4 & NMR & $1 \mathrm{qkl}$ & $(12)$ & 79-154 & $140(96)$ & & \\
\hline Rpb7 & 19.1 & 171 & 61 & & & & & & & & & \\
\hline Rpb8 & 16.5 & 146 & 43 & S. cerevisiae & Rpb8 & NMR & 1a1d & (13) & & $114(78)$ & & $\begin{array}{l}\text { Cys24, } \\
\text { Cys36 }\end{array}$ \\
\hline Rpb9 & 14.3 & 122 & 37 & Thermococcus celer & $\begin{array}{l}\text { Rpb9 COOH- } \\
\text { term. domain }\end{array}$ & NMR & 1qур & (74) & $67-112$ & $117(96)$ & $\begin{array}{l}\operatorname{Zn} 3(27.5) \\
\operatorname{Zn} 4(26.4)\end{array}$ & \\
\hline Rpb10 & 8.3 & 70 & 73 & $\begin{array}{l}\text { Methanobacterium } \\
\text { thermoautotrophicum }\end{array}$ & Rpb10 homolog & NMR & 1 ef 4 & $(87)$ & $3-55$ & $65(93)$ & $\operatorname{Zn} 1(31.9)$ & \\
\hline Rpb11 & 13.6 & 120 & 50 & E. coli & $\alpha$ & X-ray & Tbdf & (69) & $19-101$ & $110(92)$ & & \\
\hline Rpb12 & 7.7 & 70 & 43 & & & & & & & $36(51)$ & $\operatorname{Zn} 5(24.7)$ & \\
\hline Total & 513.6 & 4565 & 53 & & & & & & & $3219(83)^{\top}$ & $8+1$ & \\
\hline
\end{tabular}

${ }^{1}$ Percentage of identical amino acid residues, for Rpb1 excluding the $\mathrm{COOH}$-terminal domain. $\quad{ }^{2}$ Peaks in the zinc anomalous difference Fourier shown in Fig. 1. Peaks are numbered

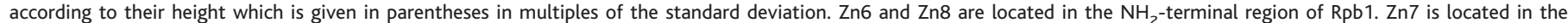

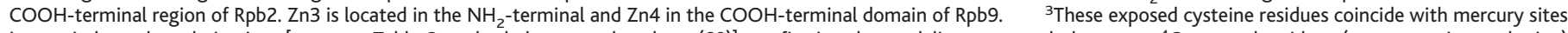

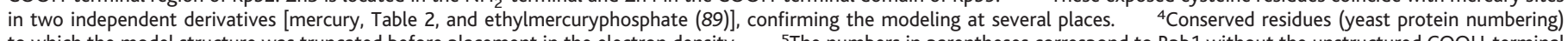

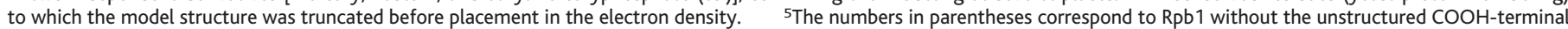

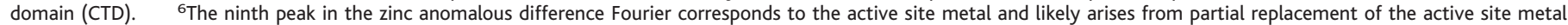
by zinc. $\quad{ }^{7}$ The number in parentheses corresponds to the pol II mutant used in structure determination, which lacks subunits Rpb4 and Rpb7. 


\section{RESEARCH ARTICLES}

terminal region of Rpb1, connected by a linker $\mathrm{COOH}$-terminal region of $\mathrm{Rpb} 2$; and (iv) crosslinking of Rpb5 to the $\mathrm{COOH}$-terminal region of Rpb1 and of Rpb3 to residues 901 to 992 of Rpb2 (39). of appropriate length; (iii) one zinc site in the
The current backbone model comprises 8 polyalanine fragments for Rpb1, 10 fragments for Rpb2, and major portions of all small subunits (Table 1). It accounts for the entire molecular volume observed in the crystals and contains 3219 residues, about $83 \%$ of the total, assuming all residues are ordered except the $\mathrm{COOH}$-terminal domain of Rpb1. Building of an atomic model is well advanced.

General architecture and DNA binding. The two largest subunits, Rpb1 and Rpb2, form distinct masses with a deep cleft between them
Fig. 2. Subunit structures determined previously or rebuilt here fitted to the experimental pol II electron density. The solventflattened MIRAS electron density map (blue) is contoured at 1.0 o. Experimental phases in the resolution range 40 to $3.1 \AA$ were used to calculate the map. In (A) and (B), the map was filtered with program MAPMAN to reduce noise (84). This map facilitated fold recognition but appears to be at lower resolution, and side chain density is largely removed. In (C), the original map is shown, which is noisi-

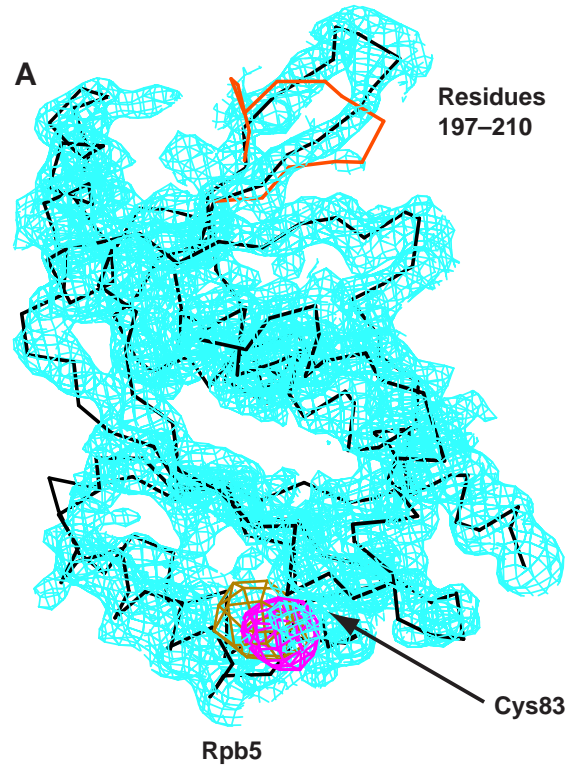
details. (A) C $\alpha$ model of Rpb5 [black (47)] fitted to the density (blue). A loop that is involved in packing against Rpb1 is in a different conformation in pol II than in the structure of free Rpb5 (orange). Peaks of anomalous difference Fourier transforms of two mercury derivatives (pink, yellow, both contoured at $5 \sigma$ ) coincide with the position of Cys83. (B) $\mathrm{C} \alpha$ traces of the NMR structure of the Rpb10 homolog from Methanobacterium thermoautotrophicum [orange (81)] fitted to the density (blue) and the rebuilt backbone model for yeast Rpb10 (black). The er but reveals many
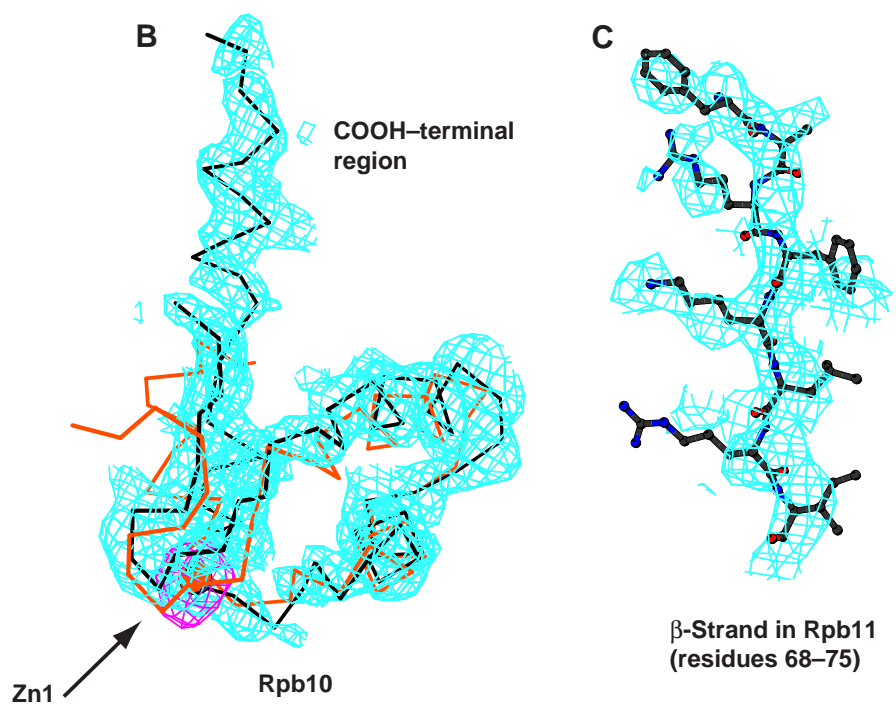

$\beta$-Strand in Rpb11 (residues 68-75) location of the zinc ion in the NMR structure coincides with a strong peak in the zinc anomalous Fourier (pink, contoured at $7 \sigma$ ). (C) One of the $\beta$ strands in Rpb11 (black, residues 68 to 75 ) fitted to the density (blue). Distinct electron density is present for several side chains. The model was obtained by placing the conserved core of $E$. coli $\alpha(69)$ and replacing the side chains with those in yeast Rpb11 using the most common rotamer. This figure was prepared with BOBSCRIPT (85) and MOLSCRIPT (86).

Table 2. Data collection and MIRAS phasing.

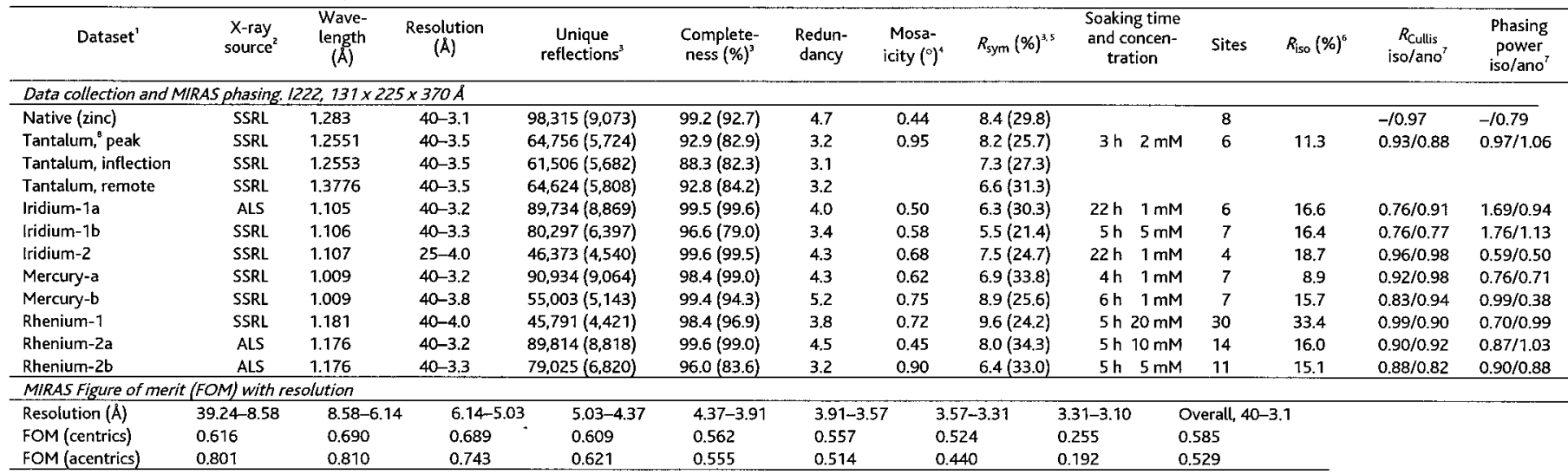

${ }^{1}$ Numbers following the element names indicate different heavy atom compounds. Lowercase letters indicate different soaking concentrations or soaking times, leading to differences

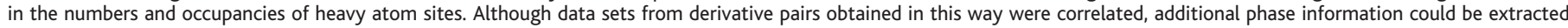

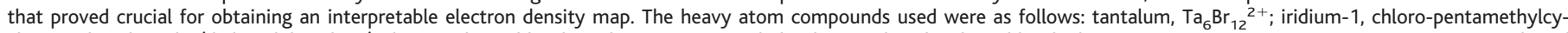

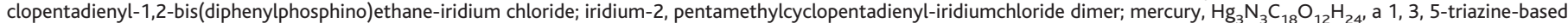

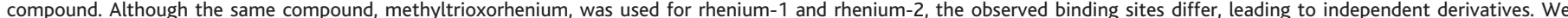

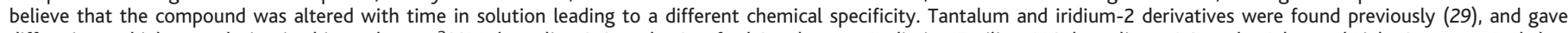

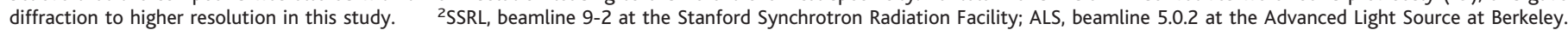

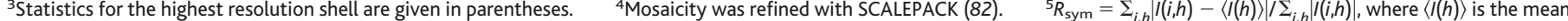

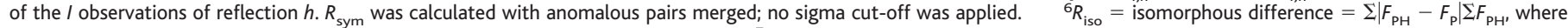

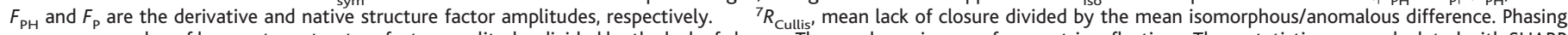

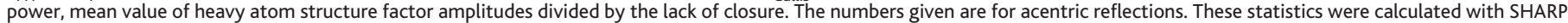

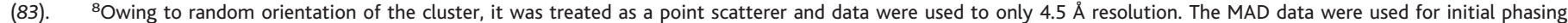
but only the peak wavelength data were used in the final MIRAS phasing. 
(Fig. 3). Each of the small subunits occurs in a single copy, arrayed around the periphery. The structure is cross-strutted by elements of Rpb1 and $\mathrm{Rpb} 2$ that traverse the cleft: A helix of $\mathrm{Rpb} 1$ bridges the cleft, and the $\mathrm{COOH}$-terminal region of $\mathrm{Rpb} 2$ extends to the opposite side. The Rpb1-Rpb2 complex is anchored at one end by a subassembly of Rpb3, Rpb10, Rpb11, and Rpb12.

The active site was located crystallographically by replacement of the catalytic $\mathrm{Mg}^{2+}$ ion with $\mathrm{Zn}^{2+}, \mathrm{Mn}^{2+}$, or $\mathrm{Pb}^{2+}$ (40). A native zinc anomalous Fourier showed a $10-\sigma$ peak that likely results from partial replacement of the active site $\mathrm{Mg}^{2+}$ by $\mathrm{Zn}^{2+}$ during protein purification (Fig. 1), and difference Fouriers obtained from crystals soaked with either $\mathrm{Mn}^{2+}$ or $\mathrm{Pb}^{2+}$ showed a single peak at the same location (41). The metal ion site occurs within a prominent loop of Rpb1 (Fig. 3), which, on the basis of preliminary sequence assignment, harbors the conserved aspartate residue motif (42). Only one catalytic metal ion was found, and only one was reported for a bacterial RNA polymerase (43), although a two-metal ion mechanism, as described for single-subunit polymerases (44), is not ruled out.

The location of duplex DNA downstream of the active site (ahead of the transcribing polymerase) was previously determined by difference 2D crystallography of an actively transcribing complex (27). Canonical B-form DNA placed in this location lies in the Rpb1-Rpb2 cleft, and can follow a straight path to the active site (Fig. 3). About 20 base pairs are readily accommodated between the edge of the polymerase and the active site, consistent with nuclease digestion studies showing the protection of about this length of downstream DNA (45). This proposal for the pol II-DNA complex is also consistent with results of protein-DNA cross-linking experiments: Rpb1 and Rpb5

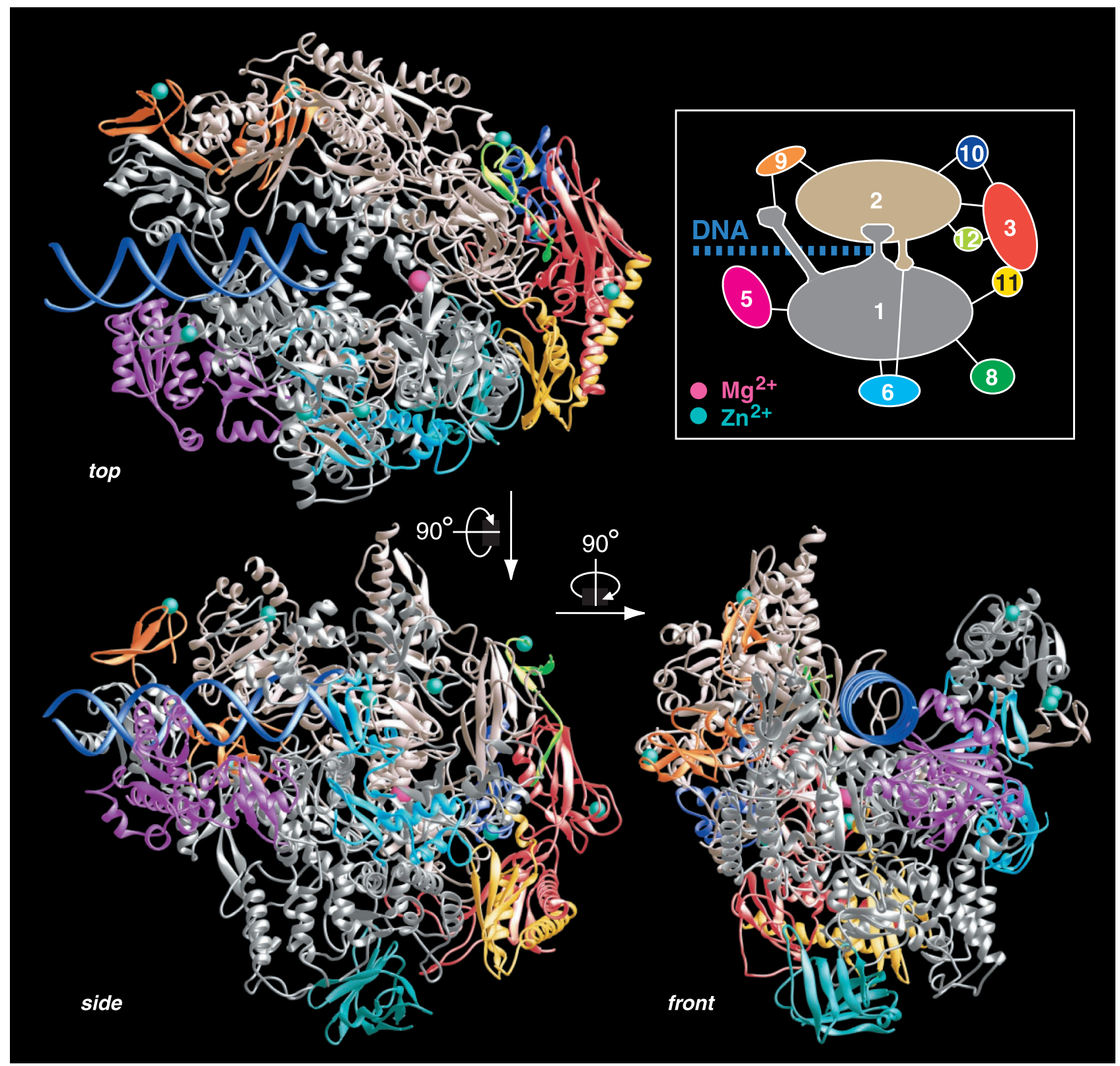

Fig. 3. Architecture of yeast RNA polymerase II. Backbone models for the 10 subunits are shown as ribbon diagrams. Secondary structure has been assigned by inspection. The three views are related by $90^{\circ}$ rotations as indicated. Downstream DNA, though not present in the crystal, is placed onto the ribbon models as 20 base pairs of canonical B-DNA (blue) in the location previously indicated by electron crys- tallographic studies (27). Eight zinc atoms (blue spheres) and the active site magnesium (pink sphere) are shown (Table 1). The box (upper right) contains a key to the subunit color code and an interaction diagram. The same views and color coding are used throughout the article. This and other figures have been prepared with RIBBONS (87). 
cross-link to one side of the DNA and Rpb2 to the other; and in the case of Rpb5, the crosslinks are located about 5 to 15 base pairs downstream of the active site (46).

Jaws position downstream DNA. Rpb5, and regions of Rpb1 and Rpb9 on the opposite side of the Rpb1-Rpb2 cleft, form "jaws" that appear to grip the DNA (Fig. 4). Both the upper and lower jaw may be mobile, opening and closing on the DNA. Mobility within Rpb5 is suggested by comparison with the x-ray crystal structure of the subunit alone (47). There was a nearly perfect fit of the subunit structure to the corresponding region of the pol II electron density map (Fig. 2A), except for a change in relative orientation of the $\mathrm{NH}_{2}$ - and $\mathrm{COOH}-$ terminal domains, and a conformational change of a loop in the COOH-terminal domain (Fig. 4B). The solvent-exposed, $\mathrm{NH}_{2}$-terminal domain (residues 1 to 142) has apparently moved by as much as $5 \AA$ in the direction of DNA in the pol II cleft, relative to the position in Rpb5 alone, with the $\mathrm{COOH}$-terminal domain (residues 143 to 215) held fixed against the body of Rpb1 (Fig. 4B). The observed position of the $\mathrm{NH}_{2}$-terminal domain in pol II is defined by crystal contacts.

\section{RESEARCH ARTICLES}

Residues in the Rpb5 loops facing the DNA are conserved (Fig. 4C). Two prolines that are strictly conserved present their side chains to the DNA with a spacing and relative orientation appropriate for contacting the DNA backbone. Proline residues have been seen to interact with backbone ribose moieties of DNA in other crystal structures (48, 49). Such nonspecific van der Waals interactions might favor a particular rotational setting of the DNA, without greatly impeding the helical screw rotation required to propel the DNA toward the active site and to unwind it for transcription.

Other conserved residues of Rpb5 are located in the linker between the $\mathrm{NH}_{2}$ - and $\mathrm{COOH}$-terminal domains and in the $\mathrm{NH}_{2}-$ terminal helix (Fig. 4C). Since the linker is not involved in subunit-subunit interactions, conserved residues might ensure a directed movement of the $\mathrm{NH}_{2}$-terminal domain. Conserved residues in the $\mathrm{NH}_{2}$-terminal helix form a positive charge cluster that is too far from DNA to contact it directly, but might attract it through long-range interactions.

$\mathrm{Rpb} 5$ is likely to play a role in transcriptional activation (50). The $\mathrm{NH}_{2}$-terminal do- main of Rpb5 binds to the transactivation domain of the hepatitis $\mathrm{B}$ virus $\mathrm{X}$ protein (51). Another Rpb5-interacting protein interferes with transactivation (52). Some activators might function by enhancing jaw-DNA interaction, thereby stabilizing transcription initiation or elongation complexes.

The upper jaw, formed by regions of Rpb1 and Rpb9, corresponds with a domain previously shown to be mobile by $2 \mathrm{D}$ crystallography (53). Rpb9 is composed of two zinc-binding domains separated by a 15 -residue linker. A stretch of the linker adds a $\beta$ strand to a sheet in the Rpb1 region of the jaw. Rpb9 therefore buttresses Rpb1, possibly constraining mobility of the jaw and strengthening its grip on DNA. Mutations in Rpb9 alter the locations of transcription start sites (54-56), which might be explained by a diminished grip on the DNA, or alternatively, by direct Rpb9-DNA interaction before entry of the DNA into the Rpb1-Rpb2 cleft.

A clamp retains DNA. A second mobile element of pol II, previously revealed by lowresolution structures and referred to as a "hinged" domain, was suggested to clamp nucleic acids in the cleft (29). This element, here

\section{A}

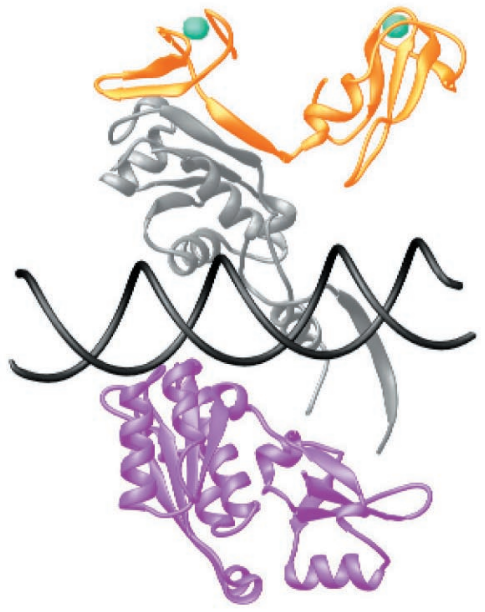

B

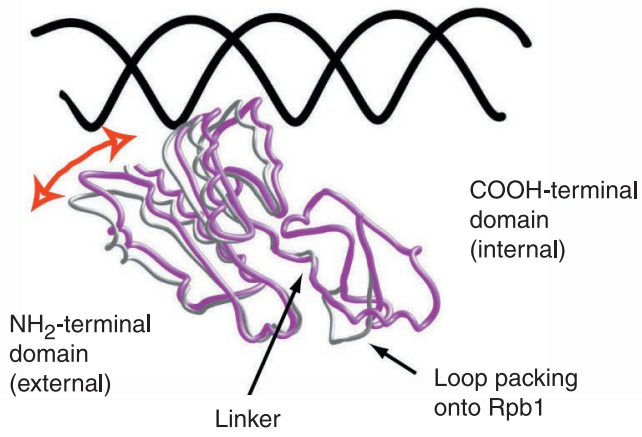

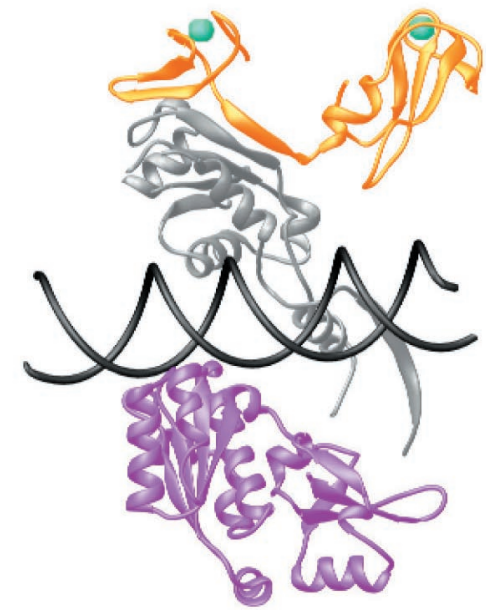

C Conserved Strictly conserved

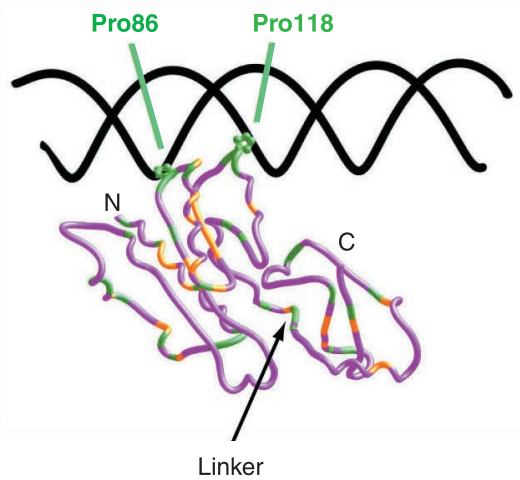

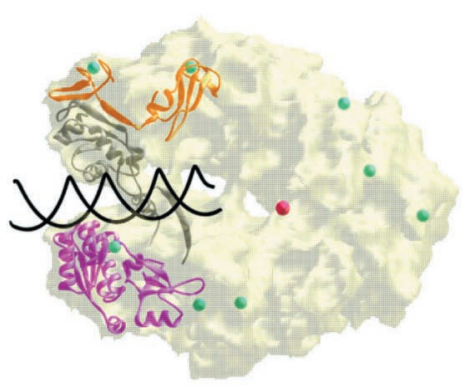

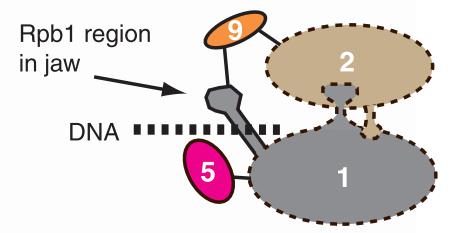

top

Fig. 4. Jaws. (A) Stereoview of structural elements constituting the jaws (left) and the location of these elements within pol II (right). (B) Mobility of the larger, $\mathrm{NH}_{2}$-terminal domain of Rpb5. Backbone models of free Rpb5 [gray (47)] and Rpb5 in pol II (pink) are shown with their smaller, $\mathrm{COOH}$-terminal domains superimposed. (C) Conservation of amino acid residues of Rpb5. 


\section{RESEARCH ARTICLES}

termed the "clamp," comprises $\mathrm{NH}_{2}$-terminal regions of Rpb1 and Rpb6 and the $\mathrm{COOH}$ terminal region of Rpb2 (Fig. 5). All three polypeptides enter at the base of the clamp near the active site, allowing a degree of conformational freedom but not unrestricted movement of the clamp. Within the Rpb6 region, 17 out of 42 residues are negatively charged, forming a cluster near the bottom of the clamp. This region of Rpb6 is also phosphorylated by casein kinase II, suggesting a regulatory role (57).

The clamp forms one side of the Rpb1$\mathrm{Rpb} 2$ cleft, where it may interact with the DNA (and the DNA-RNA hybrid, see below) from the active site to about 15 residues downstream. This DNA region corresponds with a doublestranded DNA binding site, 3 to 12 residues downstream of the active site, defined by biochemical analysis of $E$. coli RNA polymerase $(58-60)$. This binding site was referred to as a "sliding clamp" because of its importance for the great stability of a transcribing complex and processivity of transcription (60). Closure of the clamp over the DNA could account for this stability. Such a movement of the $\mathrm{NH}_{2}$-terminal region of the largest subunit was inferred from cross-linking studies of the E. coli enzyme (58). Although the clamp is seen here in an open conformation, it is involved in crystal contacts and the observed position is likely determined by the crystal lattice. The electron density in this region is of lower quality than elsewhere in the map, and the three zinc peaks associated with the region have the lowest heights (Zn6-8, Table 1), also consistent with mobility of the clamp.

DNA-RNA hybrid binding site, RNA binding site. Transcribing polymerases have been shown to harbor an unwound region of DNA, or "bubble," within which is centered a DNA-RNA hybrid of 8 or 9 base pairs, with the $3^{\prime}$ or growing end of the RNA at the active site (Fig. 6A) (60). Linear extension of duplex DNA placed in our crystallographic model, to accommodate the DNA-RNA hybrid, is impossible because of an element from Rpb2 blocking the path (Figs. 3, 4, and 6). This blocking element corresponds with a "wall" of density previously noted in the structure of bacterial RNA polymerase (43). Because of the wall, and because the active site lies well beneath the level of the downstream DNA, the DNA-RNA hybrid must be tilted relative to the axis of the downstream DNA (dashed line in Fig. 6C). The exact orientation of the hybrid remains to be determined.

At the upstream end of the DNA-RNA hybrid ( $5^{\prime}$ end of the RNA, remote from the active site), the strands must separate. Biochemical studies show that the RNA strand enters a binding site on the protein, extending from about 10 to 20 nucleotides upstream of the active site (61). There are two prominent grooves in the pol II structure exiting the hybrid binding site, each of which could accommodate

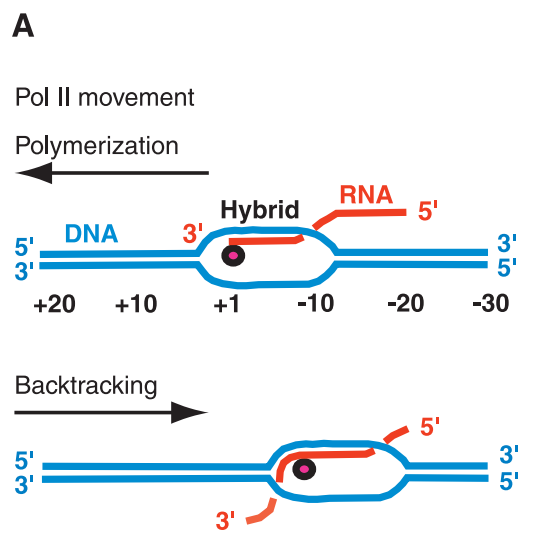

C

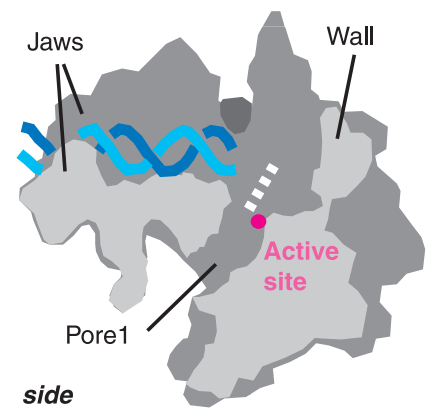
2D crystallography (25). one, but not two, nucleic acid strands. One groove winds around the base of the clamp (Fig. 7, groove 1). The other is between the

lower part of the wall and Rpb1, and continues downward between Rpb1 and Rpb11 (Fig. 7, groove 2). We favor groove 1 as the RNA
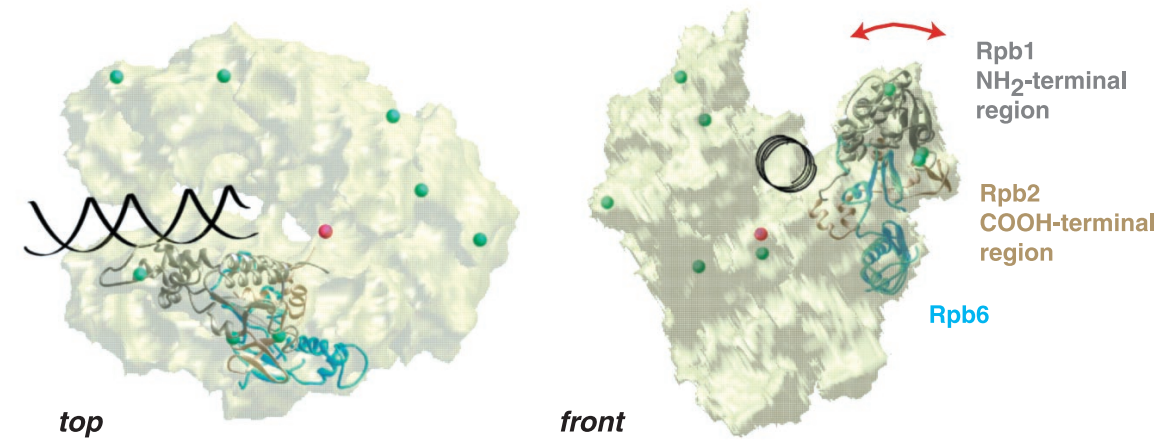

Fig. 5. Clamp. Structural elements constituting the clamp and their location in pol II are shown. The $\mathrm{COOH}$-terminal region of Rpb2 and the $\mathrm{NH}_{2}$-terminal region of Rpb1 bind one and two zinc ions, respectively (blue spheres). The $\mathrm{NH}_{2}$-terminal tail region of $\mathrm{Rpb} 6$ extends from its main body (at the bottom in the front view) into the clamp. The direction of movement of the clamp revealed by comparison with electron crystal structures (29) is indicated (double-headed red arrow).

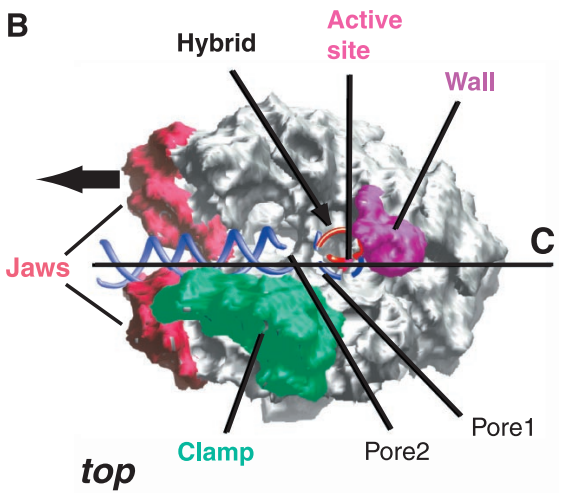

D

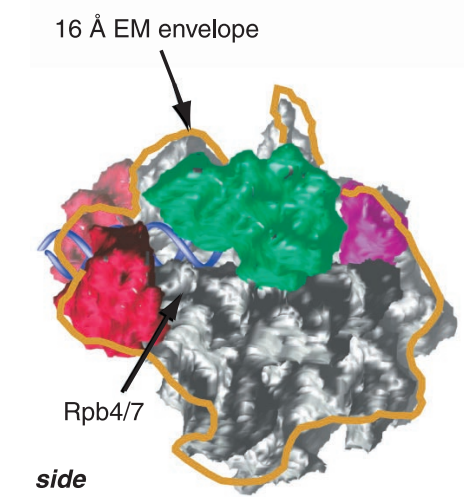

Fig. 6. Topology of the polymerizing complex, and location of Rpb4 and Rpb7. (A) Nucleic acid configuration in polymerizing (top) and backtracking (bottom) complexes. (B) Structural features of functional significance and their location with respect to the nucleic acids. A surface representation of pol II is shown as viewed from the top in Fig. 3. To the surface representation has been added the DNA-RNA hybrid, modeled as nine base pairs of canonical A-DNA (DNA template strand, blue; RNA, red), positioned such that the growing $\left(3^{\prime}\right)$ end of the RNA is adjacent to the active site metal and clashes with the protein are avoided. The exact orientation of the hybrid remains to be determined. The nontemplate strand of the DNA within the transcription bubble, single-stranded RNA and the upstream DNA duplex are not shown. (C) Cutaway view with schematic of DNA (blue) and with the helical axis of the DNA-RNA hybrid indicated (dashed white line). An opening in the floor of the cleft that binds nucleic acid exposes the DNA-RNA hybrid (pore 1) to the inverted funnel-shaped cavity below. The plane of section is indicated by a line in (B), and the direction of view perpendicular to this plane (side) is as in Fig. 3. (D) Surface representation as in (B), with direction of view as in (C). The molecular envelope of pol II determined by electron microscopy of 2D crystals at $16 \AA$ resolution is indicated (yellow line), as is the location of subunits Rpb4 and Rpb7 (arrow, Rpb4/7), determined by difference 
binding site for three reasons. First, the length and location of the groove are appropriate for binding a region of RNA 10 to 20 nucleotides from the active site, in agreement with biochemical studies. Second, the RNA path would lead back toward the downstream DNA, ending in close proximity to the $\mathrm{NH}_{2}$-terminal region of Rpb1 (defined by a zinc site). This path would accord with the reported cross-linking of RNA about 20 nucleotides upstream of the active site to the $\mathrm{NH}_{2}$-terminal region of the largest subunit of $E$. coli RNA polymerase ( $58-$ 60 ). Finally, RNA in the groove at the base of the clamp could explain the great stability of transcribing complexes. The affinity of the polymerase for the DNA template is coupled to the presence of an RNA transcript (60). We speculate that closure of the clamp over DNA, assuring its retention in a transcribing complex, would enlarge the groove at the base of the clamp, and subsequent binding of RNA in the groove would prevent the clamp from reopening. RNA would act as a lock on the closed conformation of the clamp.

Mobility of the clamp may also be modulated by interactions with other pol II subunits and transcription factors, for example, Rpb4 and Rpb7. Although these two small subunits were absent from the form of pol II analyzed here, their approximate location is known from electron microscopy of 2D crystals (25). A surface representation of the crystallographic backbone model corresponds closely with the molecular envelope from 2D crystals (Fig. 6D). On this basis, Rpb4 and Rpb7 occupy a crevice in the surface between the lower jaw and the clamp (Fig. 6D). Interaction with either of these mobile elements or with downstream DNA could underlie the requirement for Rpb4 and $\mathrm{Rpb} 7$ for the initiation of transcription (22).

\section{RESEARCH ARTICLES}

A funnel for substrate entry, backtracking, and elongation factor access. The floor of the Rpb1-Rpb2 cleft, which supports duplex DNA and the DNA-RNA hybrid, is very thin and perforated, exposing the nucleic acids to the space below. The perforation is bisected by the helix that forms a bridge between Rpb1 and Rpb2, creating two pores, one of which lies beneath the active site (pore 1) and the other, beneath the downstream DNA (pore 2). Both pores are about $12 \AA$ in diameter and lie at the apex of an inverted funnel-shaped cavity, which increases to about $30 \AA$ in diameter at the opposite side of pol II (Fig. 7, bottom). As the Rpb1-Rpb2 cleft is occupied by duplex DNA and the DNA-RNA hybrid during transcription, nucleotides may be unable to enter above the active site and may instead gain access from below, through the funnel and pore 1, as previously suggested for both pol II and bacterial RNA polymerase $(29,43)$.

The funnel and pore 1 may play similar roles in other aspects of transcription. Bacterial and eukaryotic RNA polymerases oscillate between forward (polymerization) and backward (backtracking) movement during transcription (Fig. 6A) (60). Backtracking is important for proofreading and for traversing obstacles such as DNA damage, bound proteins, or natural pause sites in the DNA. During backtracking, the polymerase and associated transcription bubble move backward along both the DNA and the RNA. The region engaged in the DNARNA hybrid retreats like a zipper, releasing the $3^{\prime}$ end of the RNA in single-stranded form, and incorporating single-stranded RNA on the $5^{\prime}$ side of the transcription bubble into the hybrid (Fig. 6A). As mentioned above for access of nucleotides to the active site during polymer- ization, duplex DNA and hybrid in the Rpb1Rpb2 cleft may block release of the 3' end of the RNA into the cleft during backtracking. Rather, as suggested for entry of nucleotides, the 3' end of the RNA may exit through the funnel and pore 1.

Backtracking beyond a certain point can result in an arrested complex, unable to reverse direction, to restore the $3^{\prime}$ end of the RNA to the active site, and to resume transcription (60). We speculate that when a certain length of RNA has been extruded by backtracking, it may interact with a site in the funnel and be trapped, preventing reversal and recovery. For recovery from arrest, cleavage of the RNA is required to generate a new $3^{\prime}$ end at the active site (60). This cleavage is achieved with the help of transcript cleavage factors $(62,63)$. The funnel and pore 1 may provide access for such factors, for example, TFIIS. A small zinc-binding domain of TFIIS has an extended $\beta$ hairpin at one end with two conserved residues that come near the active site of pol II and that are critical for RNA cleavage $(15,16,64-66)$. Also included are tryptophan and arginine side chains involved in nucleic acid binding (67, 68). Modeling shows that this domain, only $20 \AA$ in diameter, can be accommodated in pore 1 with the two conserved $\beta$ hairpin residues reaching the active site, while still leaving room for an extruded strand of RNA.

Comparison with bacterial RNA polymerase. Most information about core bacterial RNA polymerase structure comes from $\mathrm{X}$-ray diffraction studies of the $\alpha_{2}$ homodimer from E. coli (69) and the $\alpha_{2} \beta \beta^{\prime}$ polymerase from Thermus aquaticus (43). Regions of sequence similarity have been noted between $\alpha$, Rpb3, and Rpb11 (69), between $\beta$ and

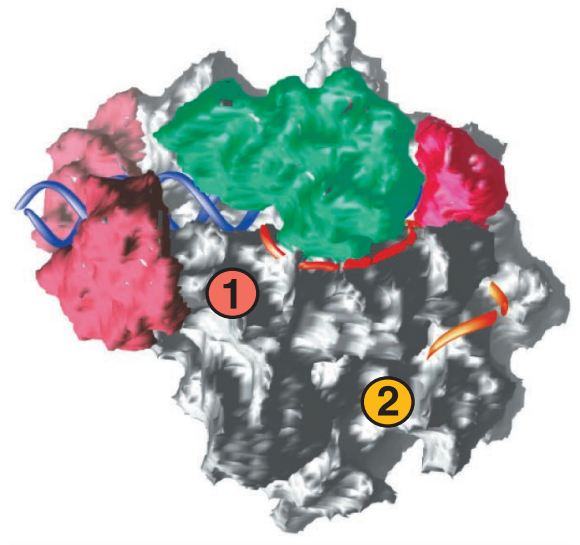

side

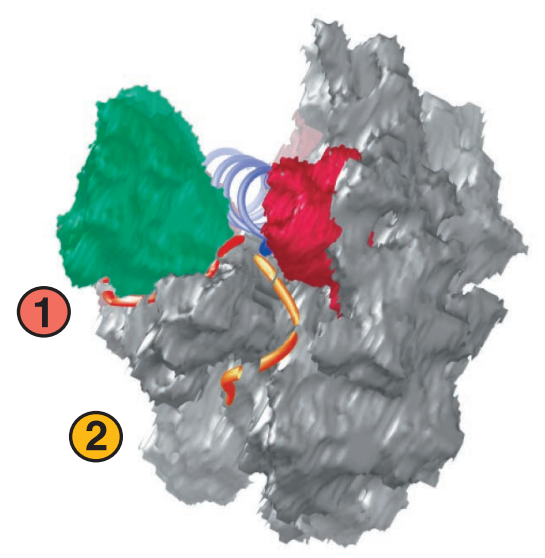

back

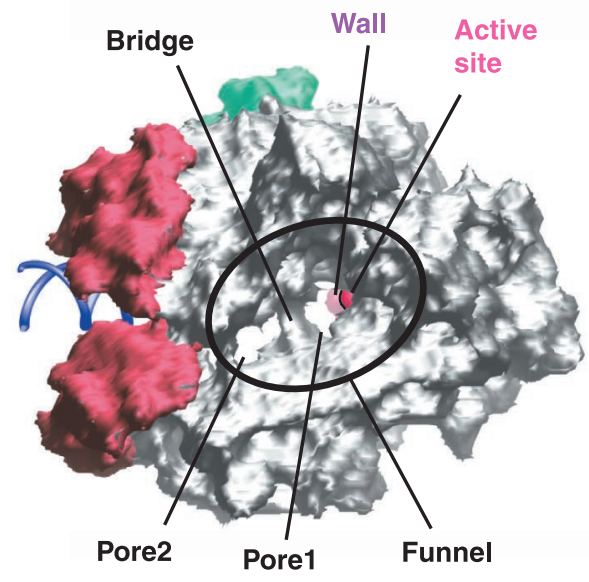

bottom
Fig. 7. Possible RNA exit grooves and funnel beneath the active site. The model of Fig. 6B is shown in two perpendicular directions of view (side, back), and also viewed from the opposite side (bottom). To the side and back views have been added dashed lines corresponding to about 10 nucleotides of RNA, lying in well-defined grooves leading away from the hybrid-binding region (groove 1, red; groove 2, orange). The nontemplate strand of the DNA within the transcription bubble and the upstream DNA duplex are not shown. To the bottom view has been added a solid line indicating the rim of the funnelshaped cavity. 


\section{RESEARCH ARTICLES}

Rpb2 (70), and between $\beta^{\prime}$ and Rpb1 (71). The crystallographic pol II model contains a conserved core of secondary structural elements similar to those in the bacterial enzyme, surrounded by divergent elements and eukaryote-specific subunits. Conserved elements are located in the vicinity of the DNARNA hybrid binding site, the adjacent downstream DNA binding site, and the sides of the funnel. Consistent with the conservation of these structural elements, similar modes of interaction with nucleic acids in the vicinity of the active site have been proposed for the eukaryotic and bacterial enzymes (72). The pore beneath the active site is conserved, and the bacterial enzyme may contain a clamp as well (73). On the other hand, the jaws, which include eukaryotespecific subunits and a domain of Rpb1, are found only in pol II, possibly reflecting their interaction with the eukaryote-specific transcription initiation factor TFIIE, as revealed by $2 \mathrm{D}$ crystallography (26). The occurrence of jaws in pol II, but not in the bacterial enzyme, presumably accounts for the nuclease protection of about 20 base pairs of downstream DNA by pol II, combacterial enzyme $(45,60)$. pared with only about 13 base pairs by the

A more detailed comparison is possible, at present, for the $\alpha_{2}$ dimer and its counterpart in pol II, the Rpb3-Rpb11 heterodimer. The $\alpha_{2}$ dimer nucleates assembly of bacterial polymerase, binding $\beta$ to form a subcomplex, which then binds $\beta^{\prime}$ to form a complete core enzyme (74). Similarly, the Rpb3-Rpb11 heterodimer binds Rpb2 to form a subcomplex (75). The location of the heterodimer in pol II is similar to that of $\alpha_{2}$ in the bacterial enzyme, and the domain conserved between Rpb3, Rpb11, and $\alpha$ exhibits an identical fold (motif of $\alpha$ helices and $\beta$ sheets forming the lower half of the subcomplex in Fig. 8). The conserved domain represents almost the entirety of Rpb11 and is responsible for Rpb3-Rpb11 interaction (or dimerization in the case of $\alpha$ ). The nonconserved domain of Rpb3 (upper half of the subcomplex in Fig. 8) interacts with the eukaryotespecific subunits Rpb10 and Rpb12. Contact of Rpb10 with Rpb3 is consistent with biochemical evidence for a stable Rpb3-Rpb11-Rpb10 subcomplex (76). Rpb12 binds through a tail, which adds a $\beta$ strand to a sheet in the nonconserved region of Rpb3. Rpb12 also interacts
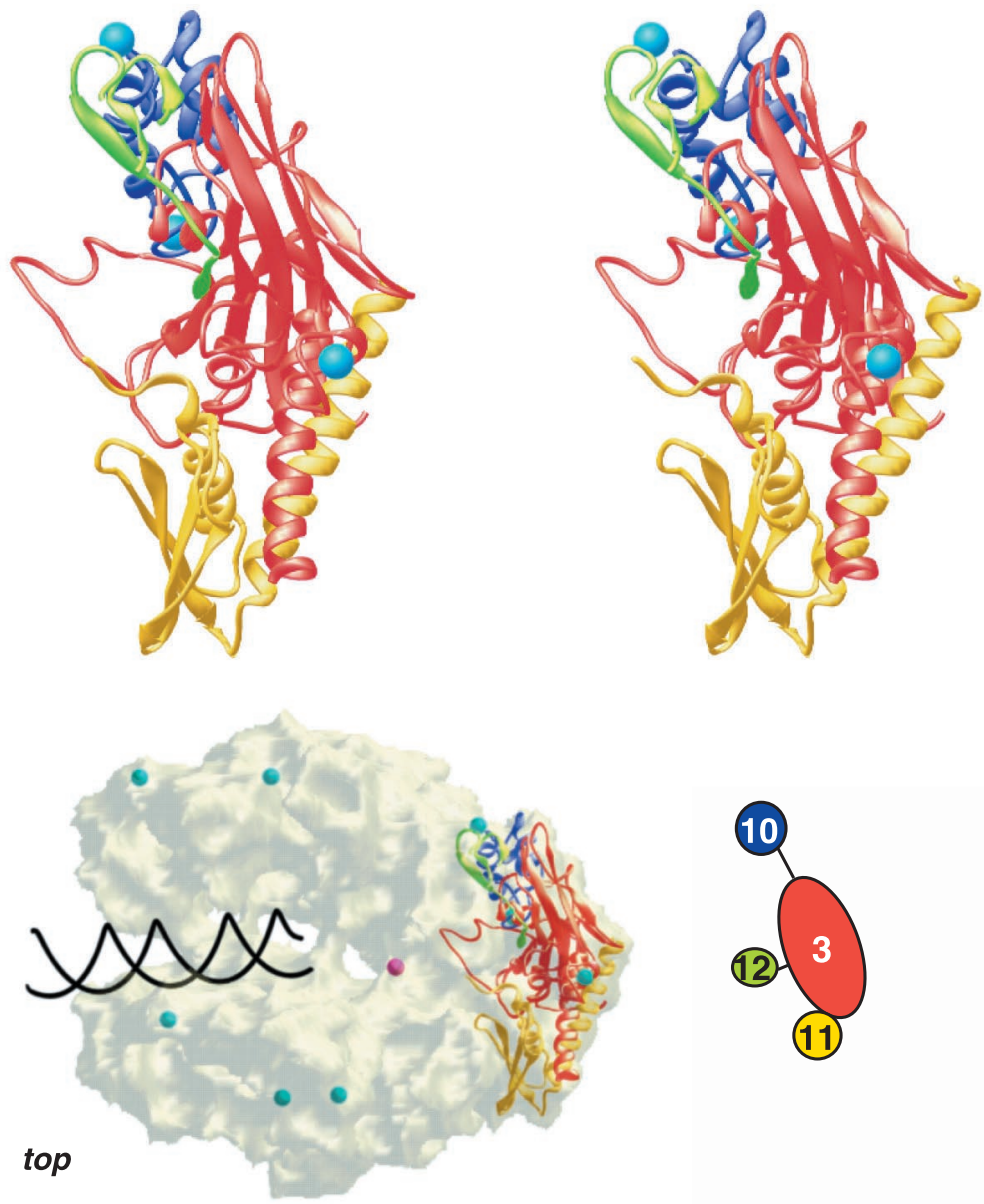

Fig. 8. The Rpb3-Rpb11-Rpb10 subcomplex and Rpb12. A stereoview of the arrangement of the four subunits is shown in the upper part, and the location of this subcomplex within pol II is shown in the lower part. with Rpb2 through its zinc-binding module. Consistent with this, Rpb12 has been shown to contact the second largest subunit in RNA polymerase I, and this interaction requires an intact zinc-binding motif (77). Moreover, a mutation in the COOH-terminal region of Rpb12 impairs assembly of RNA polymerase III (77). Thus, Rpb12 appears to play an essential role in the assembly or maintenance of all eukaryotic RNA polymerases by bridging between the Rpb3-Rpb11-Rpb10 subcomplex (or its homologs in polymerases I and III) and the second largest subunit.

Transcription pathway. The crystallographic model of pol II also gives insight into the transcription pathway and the still larger multiprotein complexes involved. The pathway begins with the formation of a TFIIB-TFIIDpromoter DNA complex and its interaction with pol II, followed by entry of TFIIE, and finally TFIIH, whose helicase activities melt DNA around the start site of transcription. The initial interaction of pol II with the promoter must be with essentially straight, duplex DNA. The pol II model, however, requires a considerable distortion for binding at the active site, which can only occur upon melting. The transition from an initial complex to a transcribing complex will therefore be accompanied by structural changes and movement of the DNA. Transcription begins with the repeated synthesis and release of short RNAs ("abortive cycling"), until a barrier at about 10 nucleotides is traversed, and chain elongation ensues. On reaching a transcript size of about 20 nucleotides, the full stability of a transcribing complex is attained. The barrier at 10 nucleotides corresponds to the point at which the $5^{\prime}$ end of the growing transcript must disengage from the template DNA and enter the proposed groove for RNA in the model. The transcript size needed for full stability corresponds with the length of RNA needed to fill the groove.

The interpretation along these lines may be extended and evaluated by the solution of pol II cocrystal structures, with the use of the pol II model for molecular replacement. Cocrystals with TFIIB and TFIIE (78) should reveal the trajectory of DNA in the initial pol II-promoter complex. Cocrystals containing pol II in the act of transcription (79) will show the locations of nucleic acids in an elongation complex. Cocrystals with TFIIS (80) may indicate the proposed exit pathway for RNA through a pore beneath the active site during backtracking. Other cocrystals may be sought to investigate the mechanism of transcriptional regulation by the multiprotein Mediator complex and associated activator and repressor proteins (4).

References and Notes

1. N. A. Woychik, Cold Spring Harbor Symp. Quant. Biol. 63, 311 (1998).

2. R. G. Roeder, Trends Biochem. Sci. 21, 327 (1996). 


\section{RESEARCH ARTICLES}

3. R. C. Conaway and J. W. Conaway, Prog. Nucleic Acid Res. Mol. Biol. 56, 327 (1997).

4. Y. J. Kim, S. Bjorklund, Y. Li, M. H. Sayre, R. D. Kornberg, Cell 77, 599 (1994).

5. G. Otero et al., Mol. Cell 3, 109 (1999).

6. J. L. Corden and M. Patturajan, Trends Biochem. Sci. 22, 413 (1997)

7. J. L. Kim, D. B. Nikolov, S. K. Burley, Nature 365, 520 (1993).

8. D. B. Nikolov et al., Nature 377, 119 (1995).

9. Y. Kim, J. H. Geiger, S. Hahn, P. B. Sigler, Nature 365 512 (1993)

10. P. Kosa, G. Ghosh, B. S. DeDecker, P. B. Sigler, Proc Natl. Acad. Sci. U.S.A. 94, 6042 (1997)

11. W. Zhu et al., Nature Struct. Biol. 3, 122 (1996).

12. F. del Rio-Portilla, A. Gaskell, D. Gilbert, J. A. Ladias, G. Wagner, Nature Struct. Biol. 6, 1039 (1999).

13. S. Krapp, G. Kelly, J. Reischl, R. O. J. Weinzierl, S. Matthews, Nature Struct. Biol. 5, 110 (1998).

14. B. Wang, D. N. Jones, B. P. Kaine, M. A. Weiss, Structure 6, 555 (1998)

15. X. Qian et al., Biochemistry 32, 9944 (1993)

16. V. K. Olmsted et al., J. Biol. Chem. 273, 22589 (1998).

17. P. E. Morin, D. E. Awrey, A. M. Edwards, C. H. Arrowsmith, Proc. Natl. Acad. Sci. U.S.A., 93, 10604 (1996).

18. A. Sentenac et al., "Yeast RNA polymerase subunits and genes," in Transcriptional Regulation, S. L. McKnight and K. R. Yamamoto, Eds. (Monogr. 22, Cold Spring Harbor Laboratory Press, Cold Spring Harbor, NY, 1992).

19. N. F. Lue and R. D. Kornberg, Proc. Natl. Acad. Sci. U.S.A. 84, 8839 (1987).

20. E. E. Uzgiris and R. D. Kornberg, Nature 301, 125 (1983).

21. A. M. Edwards et al., Proc. Natl. Acad. Sci. U.S.A. 87 2122 (1990). The key step in pol II purification was immunoaffinity chromatography with the use of a monoclonal antibody from which pol II could be released by an elevated concentration of glycerol [N. E. Thompson, D. B. Aronson, R. R. Burgess, J. Biol. Chem. 265, 7069 (1990)].

22. A. M. Edwards, C. M. Kane, R. A. Young, R. D. Kornberg, J. Biol. Chem. 266, 71 (1991)

23. S. A. Darst, E. W. Kubalek, A. M. Edwards, R. D. Kornberg, J. Mol. Biol. 221, 347 (1991)

24. S. A. Darst, A. M. Edwards, E. W. Kubalek, R. D. Kornberg, Cell 66, 121 (1991).

25. G. J. Jensen, G. Meredith, D. A. Bushnell, R. D. Kornberg, EMBO J. 17, 2353 (1998)

26. K. K. Leuther, D. A. Bushnell, R. D. Kornberg, Cell 85 773 (1996).

27. C. L. Poglitsch et al., Cell 98, 791 (1999).

28. A. M. Edwards, S. A. Darst, S. A. Hemming, Y. Li, R. D. Kornberg, Nature Struct. Biol. 1, 195 (1994).

29. J. Fu et al., Cell 98, 799 (1999).

30. Purification of yeast pol II and crystallization in the orthorhombic space group 1222 were as described [L. Myers et al., Methods Companion Methods Enzymol. 12, 212 (1997); (29)]. The crystals, containing a single pol II in the asymmetric unit, were transferred under argon/hydrogen in seven steps from harvest buffer [390 mM $\left(\mathrm{NH}_{4}\right)_{2} \mathrm{HPO}_{4} / \mathrm{NaH}_{2} \mathrm{PO}_{4}, \mathrm{PH} 6.0,16 \%$ PEC $6000,50 \mathrm{mM}$ dioxane, and $3 \mathrm{mM}$ dithiothreito (DTT)] to stabilization buffer (100 mM MES, pH 6.3, $16 \%$ PEG 6000, $350 \mathrm{mM} \mathrm{NaCl}, 17 \%$ PEG 400, $50 \mathrm{mM}$ dioxane, and $3 \mathrm{mM}$ DTT). Crystals were cooled to $4^{\circ} \mathrm{C}$ overnight and maintained at that temperature for 5 days before flash-cooling. This treatment caused shrinkage along the crystallographic a axis to $131 \AA$ extended the diffraction limit, and led to high isomorphism of the crystals. Best results were obtained with crystals of about $200 \times 150 \times 50 \mu \mathrm{m}$. Crystals were mounted at $4^{\circ} \mathrm{C}$ in nylon loops $200 \mu \mathrm{m}$ in diameter, plunged into liquid nitrogen and stored for data collection.

31. Data collection was carried out at $100 \mathrm{~K}$ using ADSC Quantum 4-CCD detectors at the tunable high brilliance undulator beamlines 9-2 at the Stanford Synchrotron Radiation Laboratory (SSRL) and 5.0.2 at the Advance Light Source (ALS) at Lawrence Berkeley National Laboratory (Table 2). All derivative data sets were collected at the anomalous peak energy of the heavy metal to maximize the anomalous signal. Care was taken to ensure completeness and reasonable redundancy in all resolution shells. Crystals were aligned with the long $c$ axis approximately along the spindle axis to minimize overlap of reflections and to record anomalous pairs within the shortest period of time. Further, overlap of reflections at a resolution higher than $4 \AA$ was avoided by using crystals with low mosaicity and $0.5^{\circ}$ oscillation steps. Diffraction data were processed with DENZO and SCALEPACK (82)].

32. For heavy atom derivatization, crystals were soaked at $4^{\circ} \mathrm{C}$ in stabilization buffer containing heavy atom compounds as indicated (Table 2). MAD phases were calculated with MLPHARE (88) using three tantalum data sets collected at the anomalous peak, inflection, and a remote wavelength (Table 2 ).

33. Single heavy atom sites were located by isomorphous and anomalous cross-difference Fourier analysis. New derivatives could best be identified in anomalous difference Fourier maps, most likely because the low signal arising from the addition of only a few heavy atoms to a large unit cell is best detected in the absence of noise resulting from nonisomorphism.

34. Standard heavy atom compounds were too reactive and destroyed diffraction beyond $6 \AA$ resolution About 600 crystals were treated with heavy atom compounds, and a total of 60 data sets were collected to obtain those used in phasing.

35. Initially, heavy atom parameters were refined and MIRAS phases were calculated with MLPHARE (88). The molecular boundary was determined by the automatic procedure implemented in DM [K. Cowtan Joint CCP4 and ESF-EACBM Newsl. Protein Crystallogr. 31, 34 (1994)], and the phases were further improved by solvent flattening and histogram matching in DM. These phases revealed lower occupancy sites that were subsequently included. Parameters were further refined and final MIRAS phases were calculated with SHARP (83) (Table 2). The resulting phases were improved by density modification with DM.

36. Electron density maps were viewed and model building carried out with the program O [T. A. Jones, J. Y. Zou, S. W. Cowan, M. Kjeldgaard, Acta Crystallogr. A47, 110 (1991)]

37. The conserved core of the E. coli $\alpha$ homodimer comprises two pairs of helices flanked by two $\beta$ sheets and is responsible for dimerization (69). This fold motif was one of the first interpretable feature of early pol II maps and was ascribed to the Rpb3Rpb11 heterodimer. Rpb3 contains, in addition, a second domain (residues 43 to 162), whose tracing was greatly facilitated by the presence of a zincbinding motif (residues 86 to 95). The location of residue Cys207 in a long loop agrees with the position of a mercury binding site (Table 1). The overal fold of the second domain is similar to that in the nonconserved domain of $E$. coli $\alpha$ although only small parts show limited sequence similarity. The core of the structure obtained by nuclear magnetic resonance (NMR) of the Rpb10 homolog from Meth anobacterium thermoautotrophicum (residues 1 to 48 of the yeast protein) (C. D. Mackereth, C. H Arrowsmith, A. M. Edwards, L. P. Mclntosh, unpublished data) could be fitted into the pol II density in close proximity to the Rpb3-Rpb11 heterodimer with a zinc atom in the model coincident with an experimental zinc peak (Fig. 2B). The $\mathrm{COOH}$-terminal region of Rpb10 could be modeled as polyalanine (Fig. $2 \mathrm{~B}$ ). Two domains in the crystal structure of yeast Rpb5 (residues 1 to 142 and 143 to 215) (F. Todone, R. O. J. Weinzierl, P. Brick, S. Onesti, unpublished data) were fitted to the electron density as rigid bodies (Fig. 2A). Except for residues 197 to 210, no rebuilding was carried out. Residue Cys83 coincided with a mercury binding site (Table 1). A backbone model of residues 51 to 59 and 78 to 155 (yeast numbering) from the NMR structure of human Rpb6 (12) was placed below the clamp. The model was corrected for a slight change in the angle between the two helices and partially rebuilt. Following continuous density, the model was extended at the $\mathrm{NH}_{2}$-terminus by residues that are flexible in free Rpb6(12), but which become ordered in pol II. The $\beta$ barrel fold of yeast Rpb8 determined by NMR (13) was easily detected at an exposed position in pol that is not involved in crystal packing. Rpb8 contains two cysteine residues (Cys24 and Cys36), which lie within one sheet. Both are derivatized by mercury compounds (Table 1), confirming the location of Rpb8 and defining the orientation of the pseudosymmetric subunit. The NMR structure of the $\mathrm{COOH}$ erminal domain of Rpb9 (14) could be placed with the aid of an experimental zinc peak. An extended density connecting to another domain that also contains an experimental zinc peak was identified as the interdomain linker. The linker length corresponds to the distance required to place the cysteines of the $\mathrm{NH}_{2}$-terminal domain at the zinc position.

38. A first backbone model included regions in all subunits and contained a total of 2753 residues. Phases calculated from this preliminary model were recombined with the experimental MIRAS phases using SIGMAA (88). The electron density map obtained by application of these combined phases and density modification showed novel features such as continuous density where chain breaks were previously encountered. This map was used to adjust and complete the backbone model. Another phase combination step resulted in a further improved map in which no errors in the backbone tracing could be detected. A total of 18 polyalanine fragments were built for Rpb1 and Rpb2, ranging in length from 13 to 666 residues. No structural information was available for the smallest subunit Rpb12, which binds zinc. After seven out of the eight zinc ions in pol II were assigned to other subunits, a distinct density could be assigned for Rpb12 located around a zinc position on the outer surface of pol II, facing away from the cleft.

39. T. Miyao, A. Honda, Z. Qu, A. Ishihama, Mol. Gen. Genet. 259, 123 (1998).

40. The active site metal ion in RNA polymerases can be replaced by $\mathrm{Mn}^{2+}$ [Y. Huang, F. Eckstein, R. Padilla, R. Sousa, Biochemistry 36, 8231 (1997)], as well as $\mathrm{Pb}^{2+}$ (43). Further, $\mathrm{Mn}^{2+}$ and $\mathrm{Zn}^{2+}$ have been used to specifically replace catalytic magnesium ions in DNA polymerases [Y. Kim et al., Nature 376, 612 (1995); S. Doublie, S. Tabor, A. M. Long, C. C. Richardson, T. Ellenberger, Nature 391, 251 (1998); L. S. Beese and T. A. Steitz, EMBO J. 10, 25 (1991); V. Derbyshire et al., Science 240, 199 (1988)]

41. A crystal was soaked overnight with $10 \mathrm{mM} \mathrm{MnCl}$ The anomalous peak of manganese at $6539 \mathrm{eV}$ is not accessible experimentally. However, the theoretical signal at the accessible x-ray energy of $9600 \mathrm{eV}$, in the tail region of the peak, amounts to about two electrons. Diffraction data were collected at this energy, below the absorption edge of zinc where the only measurable anomalous signal derives from manganese (P. Cramer et al., data not shown). The experimental phases were of sufficient quality to reveal a single peak of height $6.2 \sigma$ in anomalous difference Fouriers. Another crystal was soaked in $1 \mathrm{mM}$ $\mathrm{Pb}(\mathrm{OAc})_{2}$ for 2 hours, and diffraction data were collected at the anomalous peak wavelength for lead. The location of the $15.6 \sigma$ peak derived from these data precisely matches the manganese site.

42. E. Zaychikov et al., Science 273, 107 (1996).

43. G. Zhang et al., Cell 98, 811 (1999).

44. T. A. Steitz, Nature 391, 231 (1998).

45. G. A. Rice, J. Chamberlin, C. M. Kane, Nucleic Acids Res. 21, 113 (1993)

46. T.-K. Kim et al., Proc. Natl. Acad. Sci. U.S.A. 94 12268 (1997).

47. F. Todone, R. O. J. Weinzierl, P. Brick, S. Onesti, unpublished data.

48. C. W. Müller, F. A. Fey, M. Sodeoka, G. L. Verdine, S. C. Harrison, Nature 373, 311 (1995).

49. P. Cramer, C. J. Larson, G. L. Verdine, C. W. Müller EMBO J. 16, 7078 (1997).

50. T. Miyao and N. A. Woychik, Proc. Natl. Acad. Sci. U.S.A. 95, 15281 (1998)

51. J. H. Cheong, M. Yi, Y. Lin, S. Murakami, EMBO J. 14 143 (1995)

52. D. Dorjsuren et al., Mol. Cell. Biol. 18, 7546 (1998).

53. F. Asturias, G. Meredith, C. Poglitsch, R. Kornberg, J. Mol. Biol. 272, 536 (1997)

54. E. M. Furter-Graves, R. Furter, B. D. Hall, Mol. Cell. Biol. 11, 4121 (1991).

55. E. M. Furter-Graves, B. D. Hall, R. Furter, Nucleic Acids Res. 22, 4932 (1994). 
56. W. W. Hull, K. McKune, N. A. Woychik, Genes Dev. 9, 481 (1995).

57. K. Kayukawa, Y. Makino, S. Yogosawa, T. Tamura, Gene 234, 139 (1999).

58. E. Nudler, E. Avetissova, V. Markovtsov, A. Goldfarb, Science 273, 211 (1996).

59. E. Nudler, I. Gusarov, E. Avetissova, M. Kozlov, A. Goldfarb, Science 281, 424 (1998).

60. E. Nudler, J. Mol. Biol. 288, 1 (1999).

61. G. A. Rice, C. M. Kane, M. J. Chamberlin, Proc. Natl. Acad. Sci. U.S.A. 88, 4245 (1991).

62. M. G. Izban and D. S. Luse, Genes Dev. 6, 1342 (1992).

63. T. L. Johnson and M. J. Chamberlin, Cell 77, 217 (1994).

64. W. Powell, B. Bartholomew, D. Reines, J. Biol. Chem. 271, 22301 (1996).

65. D. E. Awrey et al., J. Biol. Chem. 273, 22595 (1998)

66. C. Jeon, H. Yoon, K. Agarwal, Proc. Natl. Acad. Sci. U.S.A. 91, 9106 (1994).

67. K. Agarwal et al., Biochemistry 30, 7842 (1991).

68. H. Yoon, A. S. Sitikov, C. Jeon, K. Agarwal, Biochemistry 37, 12104 (1998)

69. G. Zhang and S. A. Darst, Science 281, 262 (1998).

70. D. Sweetser, M. Nonet, R. A. Young, Proc. Natl. Acad. Sci. U.S.A. 84, 1192 (1987).

71. R. S. Jokerst, J. R. Weeks, W. A. Zehring, A. L. Greenleaf, Mol. Gen. Genet. 215, 266 (1989).

72. The proposed location of downstream DNA and the DNA-RNA hybrid in the crystallographic model of pol II corresponds well with that previously put forward and recently refined on the basis of protein-nucleic acid cross-linking data for the bacterial enzyme [(43) and N. Korzheva et al., manuscript submitted]. The proposed location of downstream DNA is also the same as that previously shown in $15 \AA$ electron and $6 \AA$ x-ray maps of pol II $(27,29)$, but the location of the DNA-RNA hybrid proposed here differs from that suggested on the basis of the lower resolution data. A conjecture about the location of the active site in the previous maps placed the hybrid at the downstream edge of the clamp. The active site and thus the hybrid

\title{
RESEARCH ARTICLES
}

are now definitively placed near the upstream edge of the clamp.

73. The bacterial enzyme appears to contain a counterpart of the pol II clamp, on the basis of following three observations. First, the zinc-binding $\mathrm{NH}_{2}$-terminal region of $\beta^{\prime}$ and the $\mathrm{COOH}$-terminal region of $\beta$ form a distinct structural element, just as do the corresponding regions of Rpb1 and Rpb2, respectively, in the pol II clamp. Second, these regions include motifs conserved between the bacterial and eukaryotic enzymes. Finally, the relative position of this structural element with respect to the active site and central cleft is the same in both enzymes.

74. A. Ishihama, Adv. Biophys. 14, 1 (1981).

75. M. Kimura, A. Ishiguro, A. Ishihama, J. Biol. Chem 272, 25851 (1997).

76. D. Lalo, C. Carles, A. Sentenac, P. Thuriaux, Proc. Natl. Acad. Sci. U.S.A. 90, 5524 (1993).

77. L. Rubbi, S. Labarre-Mariotte, S. Chedin, P. Thuriaux, J. Biol. Chem. 274, 31485 (1999).

78. D. A. Bushnell, K. Leuther, R. D. Kornberg, unpublished data.

79. A. L. Gnatt, J. Fu, R. D. Kornberg, J. Biol. Chem. 272, 30799 (1997).

80. A. L. Gnatt and R. D. Kornberg, unpublished data.

81. A. M. Edwards and C. Mackereth, unpublished data

82. Z. Otwinowski and W. Minor, Methods Enzymol. 276, 307 (1996).

83. E. d. la Fortelle and G. Bricogne, Methods Enzymol. B, 472 (1997).

84. G. J. Kleywegt and T. A. Jones, Acta Crystallogr. D52, 826 (1996).

85. R. M. Esnouf, J. Mol. Graphics 15, 132 (1997).

86. P. J. Kraulis, J. Appl. Crystallogr. 24, 946 (1991).

87. M. Carson, Methods Enzymol. 277, 493 (1997).

88. CCP4, Acta Crystallogr. D50, 760 (1994).

89. P. Cramer et al., data not shown.

90. For commercially unavailable heavy atom compounds, we thank P.J. Alaimo and R. Bergman (University of California, Berkeley); G. Huttner, P. Schöckers, and P. Hofmann (Universität Heidelberg); W.
Scherer and W. A. Herrmann (Technische Universität München), G. Schneider (Stockholm University); R. Huber [Max Planck Institute (MPI) Martinsried]; B. Weberndörfer and H. Werner (Universität Würzburg) and W. Jahn (MPI Heidelberg). For assistance at Stanford Synchrotron Radiation Laboratory (SSRL), beamlines 1-5, 7-1, 9-1, and 9-2, we thank H. Bellamy, A. Cohen, P. Ellis, P. Kuhn, T. McPhillips, K. Hodgson, M. Soltis, and the other members of the SSRL use support staff. This research is based in part on work done at SSRL, which is funded by the U.S. Department of Energy Office of Basic Energy Sciences. The structural biology program is supported by the $\mathrm{NIH}$ National Center for Research Resources Biomedical Technology Program and the DOE Office of Biological and Environmental Research. For help at beamline 5.0.2 of the Advanced Light Source (ALS) at Berkeley, we thank T. Earnest. We thank C. Vonrhein for help with program SHARP. We thank R. Weinzierl and S. Onesti for sending us coordinates of the Rpb5 crystal structure before publication. We thank C. D. Mackereth and L. P. Mclntosh for sending us coordinates of the Rpb10 NMR structure before publication. We thank M. Levitt, Y. Lorch, and B. Shaanan for comments on the manuscript. We gratefully acknowledge S. Darst for many contributions and for a copy of his manuscript on bacterial RNA polymerase-nucleic acid interaction before publication. P.C. was supported by a postdoctoral fellowship of the Deutsche Forschungsgemeinschaft (DFG). D.A.B. was supported by postdoctoral fellowship PF-00-014-01-GMC from the American Cancer Society. The contribution of A.L.G. was sponsored by U.S. AMRC Breast Cancer Initiative and does not necessarily reflect the policy of the government. This research was supported by $\mathrm{NIH}$ grant GM49985 to R.D.K. Coordinates of C $\alpha$ atoms are available from http://kornberg.stanford. edu and have been deposited at the Protein Data Bank (accession code 1ENO)

28 February 2000; accepted 24 March 2000

\section{Windows Through the Dusty Disks Surrounding the Youngest Low-Mass Protostellar Objects}

\author{
J. Cernicharo, ${ }^{1 *}$ A. Noriega-Crespo, ${ }^{2}$ D. Cesarsky, ${ }^{3}$ B. Lefloch, ${ }^{1,4}$ \\ E. González-Alfonso, ${ }^{1}$ F. Najarro, ${ }^{1}$ E. Dartois, ${ }^{5}$ S. Cabrit ${ }^{6}$
}

The formation and evolution of young low-mass stars are characterized by important processes of mass loss and accretion occurring in the innermost regions of their placentary circumstellar disks. Because of the large obscuration of these disks at optical and infrared wavelengths in the early protostellar stages (class 0 sources), they were previously detected only at radio wavelengths using interferometric techniques. We have detected with the Infrared Space Observatory the mid-infrared (mid-IR) emission associated with the class 0 protostar VLA1 in the $\mathrm{HH} 1-\mathrm{HH} 2$ region located in the Orion nebula. The emission arises in three wavelength windows (at 5.3, 6.6, and 7.5 micrometers) where the absorption due to ices and silicates has a local minimum that exposes the central part of the young protostellar system to mid-IR investigations. The mid-IR emission arises from a central source with a diameter of 4 astronomical units at an averaged temperature of $\sim 700 \mathrm{~K}$, deeply embedded in a dense region with a visual extinction of 80 to 100 magnitudes.

Our lack of knowledge of star formation processes led to an empirical classification of the evolutionary phases of low-mass protostars into four classes: 0, I, II, and III. These describe the amount of material available for accretion versus the mass of the central object, providing the evolutionary status of the system (1-3). Class 0 objects are the young- est protostars; they are surrounded by large and dusty envelopes that feed the central objects and their protoplanetary disks. These sources undergo violent ejection of matter related to accretion processes. The shockwaves created when the protostellar ejecta collides with the surrounding gas produce the Herbig-Haro $(\mathrm{HH})$ jets observed at optical wavelengths. These jets seem to drive the bipolar molecular outflows (4-6) detected around protostars and represent a second mass loss-driven phenomenon taking place during the earliest evolutionary stages of the

${ }^{1}$ Consejo Superior de Investigaciones Científicas, Instituto de Estructura de la Materia, Departamento Física Molecular, Serrano 121, 28006 Madrid, Spain. ${ }^{2}$ Space Infrared Telescope Facility (SIRTF) Science Center, California Institute of Technology, Pasadena, CA 91125, USA. ${ }^{3}$ Institut d'Astrophysique Spatiale, Bât. 121, Université de Paris XI, 94500 Orsay Cedex, France. ${ }^{4}$ Observatoire de Grenoble, Domaine Universitaire de Grenoble, 414 rue de la Piscine, 38406 St. Martin d'Hères, France. 5 Institute de Radioastronomie Millimétrique, Domaine Universitaire de Grenoble, 300 rue de la Piscine, 38406 St. Martin d'Hères, France. ${ }^{6}$ Départament d'études de la Matière en Infrarouge et Millimètrique, UMR 8540 du CNRS, Observatoire de Paris, 61 Av. de l'Observatoire, F-75014 Paris, France.

*To whom correspondence should be addressed. Email: cerni@astro.iem.csic.es 